\title{
Efecto del consumo de leche fermentada con Bifidobacterium y Lactobacillus casei sobre sobre ganancia de peso y duración de diarreas en cabritos lactantes
}

Deymer Antonio Gómez-Gómez, Esp. ${ }_{1}{ }_{3}$, Martha Yurani Pico-Gutiérrez, MVZ ${ }_{1}$ ' Bernardo de Jesús Marín-Mejía, M.Sc. ${ }_{2}$, Lorenzo Álvarez-Ramírez ${ }^{\star}$ Ph.D. ${ }_{2}$

${ }_{1}$ Facultad de Medicina Veterinaria y Zootecnia, Universidad Cooperativa de Colombia, Colombia. ${ }_{2}$ Facultad de Medicina Veterinaria y Zootecnia, Universidad Nacional Autónoma de México, México. ${ }_{3}$ Facultad de Medicina Veterinaria y Zootecnia, Universidad de Ciencias Aplicadas y Ambientales, Bogotá, Colombia.

Recibido: 10 de abril del 2019

Aprobado: 22 de julio del 2019

*Autor de correspondencia: Lorenzo Álvarez Ramírez, Facultad de Medicina Veterinaria y Zootecnia, Universidad Nacional Autónoma de México, Ciudad de México, México, Ciudad Universitaria, Av. Universidad \#3000, Colonia, C.U., 04510, 4142918100 (1049). Correo electrónico: alorenzo@unam.mx

Cómo citar este artículo: Gómez-Gómez DA, Pico-Gutiérrez MY, Marín-Mejía BJ, Álvarez-Ramírez L. Efecto del consumo de leche fermentada con Bifidobacterium y Lactobacillus casei sobre sobre ganancia de peso y duración de diarreas en cabritos lactantes. Spei Domus. 2017; 13(26):1-8. doi: https://doi.org/10.16925/2382-4247.2017.01.01

Resumen. Introducción: el uso de probióticos es una práctica orientada a mejorar el desempeño productivo y la salud de los animales. El objetivo del estudio fue conocer el efecto del consumo de leche fermentada con Bifidobacterium y Lactobacillus casei sobre el desarrollo de cabritos durante la lactancia artificial en términos de ganancia diaria de peso, comparado con el consumo de leche fresca. Metodología: treinta y ocho cabritos se asignaron al azar en dos grupos experimentales, alimentados con leche fresca ( $(\mathrm{Fr}, \mathrm{n}=20$ ) o leche fermentada ( $\mathrm{LFe}, \mathrm{n}=18$ ) obtenida con un cultivo de Bifidobacterium y L. casei. El destete se realizó con 56 días de edad y se estableció el día en el cual triplicaron su peso al nacimiento. Se determinó el consumo diario de leche, la ganancia diaria de peso (GDP), el número de días requerido para triplicar el peso al nacimiento y la incidencia de diarreas y su duración. Resul-

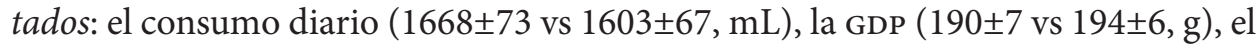
tiempo para triplicar el peso al nacimiento y la incidencia de diarreas (55 vs $60 \%$ ) no fueron diferentes entre grupos ( $p>0,05$, LFe y LFr respectivamente). La duración de las diarreas en el grupo LFr tuvo una duración mayor a 4 días en el $91 \%$ de los casos, y de $50 \%$ en el grupo LFe $(p=0,06)$. Conclusiones: bajo las condiciones del estudio, el consumo de leche fermentada con Bifidobacterium y Lactobacillus casei no influyó de manera significativa sobre el desarrollo corporal de cabritos durante la lactancia artificial, sin embargo, las diarreas fueron de menor duración.

Palabras clave: crecimiento de cabritos, lactancia artificial, leche fermentada, probióticos. 


\title{
Effect of Fermented Milk (Bifidobacterium, Lactobacillus casei) on Goat Kids Body Development
}

\begin{abstract}
Introduction: Using probiotics is a practice that tries to improve the productive performance the health of the animals. The aim of the study was to know the effect of the consumption of fermented milk with Bifidobacterium and Lactobacillus casei on the development of goat kids during artificial rearing and to compare it with the consumption of fresh non-fermented milk. Materials and methods: thirty-eight kids were randomly assigned in two experimental groups, fresh milk (FrM, $\mathrm{n}=20$ ) or fermented milk (FeM, $\mathrm{n}=18$ ) obtained with a culture of Bifidobacterium and L. casei. Weaning was at 56 days of age and having tripled the weight at birth. The daily milk consumption, the average daily weight gain (ADG), the number of days required to triple the birth weight and the incidence and duration of diarrhea were determined. Results: Daily consumption (1668 \pm 73 vs $1603 \pm 67, \mathrm{~mL}), \mathrm{ADG}(190 \pm 7$ vs $194 \pm 6$, g), the time to triple the weight at birth, and the incidence of diarrhea (55 vs $60 \%$ ) was not different between groups ( $p>0,05$, FeM and FrM respectively). Ninety-one percent of the diarrheas lasted longer than 4 days in the FrM group, and $50 \%$ in the FeM group $(p=0,06)$. Conclusions: It is concluded that under the conditions of the study, the consumption of milk fermented with Bifidobacterium and Lactobacillus casei, did not affect body development of kids during the artificial rearing, however, diarrheas were of less duration.
\end{abstract}

Keywords: artificial rearing, fermented milk, growth of kids, probiotics.

\section{Efeito do consumo de leite fermentado com Bifidobacterium e Lactobacillus casei sobre ganho de peso e duração de diarreias em cabritos lactantes}

Resumo. Introdução: o uso de probióticos é uma prática orientada para melhorar o desempenho produtivo e a saúde dos animais. O objetivo do estudo foi conhecer o efeito do consumo de leite fermentado com Bifidobacterium e Lactobacillus casei sobre o desenvolvimento de cabritos durante a lactância artificial em termos de ganho diário de peso, comparado com o consumo de leite fresco. Metodologia: 38 cabritos escolhidos aleatoriamente foram divididos em dois grupos experimentais: alimentados com leite fresco ( $\mathrm{LFr}, \mathrm{n}=20$ ) ou com leite fermentado ( $\mathrm{LFe}, \mathrm{n}=18$ ) obtido com um cultivo de Bifidobacterium e L. casei. O desmame foi realizado com 56 dias de idade e estabeleceu-se o dia no qual triplicaram seu peso de nascimento. Determinou-se o consumo diário de leite, o ganho diário de peso (GDP), o número de dias requerido para triplicar o peso de nascimento e a incidência de diarreias e sua duração. Resultados: o consumo diário (1668 \pm 73 vs $1603 \pm 67, \mathrm{~mL}$ ), a GDP ( $190 \pm 7$ vs $194 \pm 6, \mathrm{~g}$ ), o tempo para triplicar o peso de nascimento e a incidência de diarreias (55 vs $60 \%$ ) não foram diferentes entre grupos ( $\mathrm{p}>0,05$, LFe e LFr, respectivamente). A duração das diarreias no grupo LFr teve uma duração superior a 4 dias em $91 \%$ dos casos, e de $50 \%$ no grupo LFe ( $\mathrm{p}=0,06)$. Conclusões: sob as condições do estudo, o consumo de leite fermentado com Bifidobacterium e Lactobacillus casei não influenciou de maneira significativa no desenvolvimento corporal de cabritos durante a lactância artificial; no entanto, as diarreias foram de menor duração.

Palavras-chave: crescimento de cabritos, lactância artificial, leite fermentado, probióticos. 


\section{Introducción}

La cría de cabras es una de las actividades más relevantes en el sector pecuario en Latinoamérica. Se estima que en México existen 500 mil unidades de producción con esta especie animal [1]. En los sistemas lecheros, la cría artificial del cabrito, sin contacto con la madre, se utiliza regularmente para aumentar el control sobre su consumo de leche, y las condiciones higiénicas relacionadas con su contacto con enfermedades importantes en la especie [2]. Durante la lactancia del cabrito, resulta de primordial importancia que su desarrollo se mantenga creciente para alcanzar un peso determinado a la edad en que debe ser destetado o enviado al mercado. El momento del destete puede variar en la industria en función del objetivo específico del sistema de producción, pero algunos criterios generales son que el cabrito debe haber alcanzado al menos $9 \mathrm{~kg}$ de peso corporal, contar con aproximadamente 8 semanas de edad y ser capaz de consumir alimento sólido [3]. Cualquier estrategia de manejo que ayude a garantizar el alcance de estos criterios es considerada como útil para mejorar los resultados económicos de la granja.

Los probióticos se han definido como microorganismos vivos que cuando son administrados en cantidades adecuadas traen beneficios para la salud de quien los consume [4]. Una variedad importante de probióticos ha demostrado tener efectos como promotor del crecimiento, además de proteger contra infecciones intestinales que generan diarrea [4-6]. Por ejemplo, Adams et al. [7] encontraron que al administrar Propionibacterium jensenii a becerras lactantes, estas mejoraron su peso corporal. Mudgal y Baghel [8] incrementaron la ganancia de peso de crías de búfalo con suplementos de Lactobacillus acidophilus. Otros autores han descrito efectos positivos del consumo de Saccharomyces cerevisiae en el desarrollo y la salud de becerras lecheras [9]. En cabritos lactantes sin embargo, la suplementación con probióticos como Streptococus salivarius y Candida pintolopesii no ha mejorado indicadores como ganancia de peso, consumo alimenticio e incidencia de diarreas [10].

En la crianza de animales jóvenes, las enfermedades diarreicas son un problema central por sus consecuencias económicas. De constatarse su eficacia, el uso de diferentes probióticos podría representar un medio útil para prevenir pérdidas durante la lactancia, sin recurrir al uso de fármacos costosos y sin consecuencias ambientales negativas [5], [11].

Una estrategia de alimentación que incorpore probióticos podría disminuir la presentación de afecciones gastrointestinales y, por ende, mejorar los resultados en el desarrollo corporal. El objetivo del presente trabajo fue evaluar el efecto del consumo de leche fermentada con Bifidobacterium y Lactobacillus casei sobre el desarrollo corporal en términos de ganancia diaria de peso, de cabritos en un sistema de lactancia artificial.

\section{Materiales y métodos}

El estudio se realizó en el Centro de Enseñanza, Investigación y Extensión en Producción Animal en Altiplano de la Facultad de Medicina Veterinaria y Zootecnia de la Universidad Nacional Autónoma de México, localizado en Tequisquiapan, Querétaro $\left(20^{\circ} 40^{\prime} 12^{\prime \prime} \mathrm{N}, 99^{\circ} 54^{\prime} 00^{\prime \prime} \mathrm{W}\right)$, México.

Treinta y ocho cabritos (alpino francés, saanen, toggenburg) fueron retirados de su madre al momento de nacer, fueron secados, se pesaron antes de su primer alimento y se le ofreció calostro a libre acceso a las 0, 6 y 12 horas de edad. Desde el primer día y hasta el día 3 de edad, todas las crías se alimentaron de leche entera de cabra en dos tomas al día.

A partir del día 4 de edad, las crías fueron asignadas al azar a uno de los dos grupos experimentales, balanceados por raza. El grupo leche fresca ( $L F r, n=20$ ) se alimentó con leche fresca entera de cabra, el grupo de leche fermentada (LFe, $\mathrm{n}=18$ ) recibió leche entera de cabra previamente fermentada con la mezcla de probióticos comerciales de Bifidobacterium (Activia ${ }^{\oplus}$ y Lactobacillus casei $\left(\right.$ Yakult $\left.^{\star}\right)$.

A partir del día 4 y hasta el día 45 de edad, los cabritos recibieron ad libitum únicamente el tipo de leche asignada de acuerdo con su tratamiento, en dos horarios al día (08:00 y 16:00 h). Se registró la cantidad consumida por cada cabrito en ambos grupos en cada toma para obtener el total ingerido al día durante todo el estudio. El suministro de la leche se realizó mediante mamilas individuales graduadas, puestas sobre una plataforma de alimentación colectiva a una altura apropiada a la alzada de los cabritos. Durante la toma, cada animal tuvo 
acceso único a su propia mamila y la alimentación terminaba cuando perdía el interés por el amamantamiento luego de dos ofrecimientos más, ello fue decidido siempre por el mismo personal.

Los animales fueron alojados en dos corrales colectivos con un área de al menos $0,5 \mathrm{~m}^{2} /$ cabrito, provisto con techo y paredes laterales para protección de vientos, además de un espacio abierto en el que podían ejercitarse, cama seca y agua limpia de bebida. El corral disponía de comederos portátiles para la administración de forraje (alfalfa) y concentrado preiniciador con un mínimo de $19 \%$ de proteína cruda desde los 15-20 días de edad, y estuvieron separados solo para su alimentación líquida No se registró el consumo de alimento sólido.

A partir del día 46 y hasta el día 50 de edad, cuando triplicaron el peso al nacimiento, los cabritos entraron a un programa de destete parcial, en el cual el consumo diario se redujo en un $33 \%$. A partir del día 51 y hasta el día 55, el consumo se limitó a solo la mitad de la cantidad previa, y se aportó en una sola toma por la mañana. El retiro total de la leche se realizó como mínimo a los 56 días de edad y cuando su peso corporal alcanzó al menos tres veces el del nacimiento.

Todos los animales fueron pesados con una frecuencia de 8 días para determinar la ganancia diaria de peso (GDP) durante el estudio. Se calculó además el número de días requerido por cada animal para triplicar su peso al nacimiento utilizando GDP acumulada al final del estudio.

En cada alimentación, se registró el número de crías con signos de diarrea (restos de excremento líquido adherido en zona perianal) para obtener el porcentaje de animales con dicha alteración en cada grupo durante el estudio. El registro se hizo todos los días durante el periodo de estudio para estimar la duración del padecimiento en cada cabrito afectado. Cuando se presentaron casos de diarrea, se intensificaron las medidas de higiene en especial limpieza del corral y de los equipos. También se redujo en un $20 \%$ el suministro de leche a los animales enfermos, hasta su recuperación. No se utilizó tratamiento farmacológico.

Se tomó una muestra de la leche administrada (no fermentada y fermentada) con una frecuencia de cada semana y se determinó su composición mediante un analizador para leche (Foss Analytical ${ }^{\oplus}$, Milkoscan Minor). En la evaluación se registró el contenido de grasa $(\mathrm{G}, \%)$, proteína (P, $\%)$, lactosa (L, \%) y sólidos no grasos (sNG, \%).
En las muestras de la leche fermentada se registró también la acidez (g de ácido láctico/L), por medio de titulación universal [12].

\section{Preparación de la leche fermentada}

La cantidad de leche estimada para el consumo diario del grupo LFe se inoculó con $4 \%$ de una mezcla 1:1 de probióticos comerciales de Bifidobacterium y Lactobacillus casei. La leche inoculada se mantuvo a temperatura ambiente $\left(18-25^{\circ} \mathrm{C}\right)$ y bajo sombra hasta el momento de su consumo, entre 15 y 20 horas después.

\section{Análisis}

La información fue analizada mediante la técnica GLM, Tukey, $X^{2}$ y la prueba exacta de Fisher utilizando la paquetería SAs (v9). Los resultados se expresan como media $\pm \mathrm{EE}$ a menos que se indique otra cosa.

\section{Resultados}

El peso al nacimiento no fue diferente entre grupos $(2,9 \pm 0,1$ y $3,0 \pm 0,1, p>0,05$, LFr y LFe, respectivamente, $\mathrm{kg}$ ).

No se encontraron diferencias estadísticas $(p>0,05)$ en la composición de los tipos de leche utilizada (tabla 1).

Tabla 1. Composición fisicoquímica de la leche usada en ambos grupos (G, grasa; P, proteína; L, lactosa; SNG, sólidos no grasos)

\begin{tabular}{cccccc}
\hline & G & P & L & SNG & Acidez \\
\hline $\begin{array}{c}\text { L. } \\
\text { Fermentada }\end{array}$ & $3,6 \pm 0,2$ & $3,5 \pm 0,1$ & $4,2 \pm 0,1$ & $8,8 \pm 0,2$ & $87,7 \pm 7$ \\
\hline L. Fresca & $3,5 \pm 0,2$ & $3,5 \pm 0,1$ & $4,4 \pm 0,1$ & $9,0 \pm 0,2$ & - \\
\hline
\end{tabular}

Fuente: elaboración propia

El consumo diario promedio no fue diferente entre grupos (figura 1) durante el estudio. El grupo LFe tuvo un consumo total promedio de $1668 \pm 73$ y el grupo LFr de $1603 \pm 67$ por día $(\mathrm{mL}, p>0,05)$. 




Figura 1. Consumo promedio diario de leche fermentada (LFe) y leche fresca ( $\mathrm{LFr}$ ) durante la lactancia. La flecha cerrada indica reducción en el consumo debido a un brote de diarreas. Las flechas abiertas indican las reducciones intencionales como parte del destete.

Fuente: elaboración propia

El comportamiento del peso corporal no fue diferente entre grupos durante el estudio (figura 2). El tiempo requerido para triplicar el peso al nacimiento $(36,3 \pm 2$ vs $36,1 \pm 1,9$ d) y la GDP $(190 \pm 7$ vs $194 \pm 6 \mathrm{~g})$ tampoco fueron diferentes entre grupos (LFe y LFr respectivamente, $p>0,05$ ). La figura 3 muestra el comportamiento semanal de la GDP en ambos grupos.

Los grupos no difirieron en la incidencia de diarreas $(p>0,05)$ pero sí en su duración $(p<0,05)$; en el grupo LFe se observó diarrea en el $55 \%$ de los cabritos (10 animales), mientras que en el grupo LFr el padecimiento se observó en el $60 \%$ de los animales (12 cabritos). En el grupo LFr, el $91 \%$ de las diarreas tuvo una duración mayor a los 4 días, mientras que en el grupo LFe solo el 50 \% tardó más de 4 días en recuperarse $(p=0,06)$. No fue identificada la etiología de posibles agentes asociados a la diarrea.

\section{Discusión}

El presente trabajo evaluó los efectos del consumo de leche fermentada en el desarrollo corporal de



Figura 2. Peso corporal semanal en ambos grupos de acuerdo al consumo de leche fermentada (LFe) o fresca (LFr)

Fuente: elaboración propia

cabritos lactantes y los comparó con el consumo de leche fresca. Bajo las condiciones en que se realizó el estudio, el consumo de leche fermentada con Bifidobacterium y Lactobacillus casei no influyó de manera significante en el desarrollo corporal de cabritos durante la lactancia artificial. El peso 
corporal tuvo una tendencia ascendente similar en ambos grupos durante todo el estudio, y todos los animales alcanzaron el peso exigido al momento del destete. Con resultados similares a los de este estudio y usando una mayor variedad de probióti$\cos$ (L. plantarum, L. delbrueckii, L. acidophilus, $L$. rhamnosus, Bifidobacterium bifidum, Streptococcus salivarius, S. facium, Aspergillus oryza y Candida pintolopesii), otros autores no encontraron efectos significantes en cuanto a ganancia de peso, consumo e incidencia de diarreas en cabritos al final de su lactancia [10]. En corderos lactantes, Saleem et al. [13] tampoco encontraron efectos significantes de probióticos a base de Pediococcus acidilactici y P. pentosaceus. Del mismo modo, los cabritos que recibieron probióticos a base de Lactobacillus, Lactococcus, Bifidobacterium, Streptococcus, Aspergillus y Candida durante la lactancia y luego del destete no mostraron consumo alimenticio ni desarrollo corporal diferente al de los animales control [14], [15].



Figura 3. Ganancia diaria de peso (GDP) semanal en ambos grupos de acuerdo al consumo de leche fermentada (LFe) o fresca (LFr)

Fuente: elaboración propia

El uso de probióticos tiene como finalidad deseada la modificación de la población bacteriana digestiva, que da paso a la colonización de microorganismos benéficos que podrán limitar la actividad de otros patógenos [10], lo que se debería reflejar en mejoras en el consumo y conversión alimenticia, salud y desarrollo corporal. Una cantidad importante de publicaciones registra una mejora significante en el desarrollo corporal de becerras asociada al consumo de probióticos [4], [6]. En este estudio el consumo de leche fermentada con Bifiodbacterium y L. casei durante la lactancia no se asoció con un mejor desarrollo corporal.

La eficacia de un probiótico depende de la capacidad de adaptación de los microorganismos y su habilidad para competir con patógenos del tracto digestivo [14]. La dosis y variedad de microorganismos en el probiótico parecen importantes y deberán ser evaluadas a profundidad para determinar su utilidad real en cabras. En otros estudios, aunque se logró incrementar la población del microorganismo probiótico en heces, circunstancia que sugiere una colonización intestinal exitosa, los indicadores de consumo alimenticio y desarrollo corporal de becerras lecheras no se modificaron [16].

Aunque la incidencia total de diarreas no fue diferente entre grupos, los animales que recibieron la leche fermentada presentaron cuadros más cortos. Otros autores han descrito un efecto protector de L. casei contra la adhesión intestinal de E. coli, una de las principales causas de diarreas en animales jóvenes, en corderos [17] y cerdos [18], que minimizan los signos clínicos y reducen los hallazgos anatomo-patológicos. Esto coincide con nuestro resultado, también se ha descrito una menor duración de los cuadros diarreicos en becerras a las que se administró L. acidophilus [19] o Bacillus amyloliquefaciens [20]. Renaud et al. [21] por su parte, encontraron que la administración de un probiótico que contenía Pediococcus acidilactici, Enterococcus faecium, Lactobacillus acidophilus, Lactobacillus casei y Bifidobacterium bifidum aceleraba la solución de los cuadros diarreicos en comparación con lo visto al administrar un placebo. En esta misma dirección, un estudio sistemático de revisión, incluyendo docenas de ensayos [22], ha concluido que el uso de probióticos tiene la capacidad de reducir la duración de los cuadros diarreicos en humanos. Usando L. casei en combinación con un prebiótico. Ayala-Monter et al. [23] incrementaron la ganancia diaria de peso y el peso al destete, al tiempo que redujeron la incidencia de diarreas en corderos lactantes. En cabritos, sin embargo, el uso aislado del mismo probiótico no ha tenido efectos significativos [24].

Aunque no se identificó la posible etiología asociada a la diarrea, se informa el carácter endémico de agentes coliformes en este rebaño. 


\section{Conclusión}

En cabritos de razas lecheras durante la lactancia, el consumo de leche fermentada mediante el uso de Bifidobacterium (Activia ${ }^{\circledR}$ y L. casei (Yakult ${ }^{\oplus}$ ) no se asoció con un mejor desarrollo corporal, pero los cuadros diarreicos que se presentaron tendieron a durar menos, lo que podría sugerir un efecto protector benéfico a nivel digestivo.

\section{Referencias}

[1] Cuéllar JA, Tórtora J, Trejo A, Roman P. La producción caprina mexicana. Particularidades y complejidades. Ciudad de México: Ariadna Editorial; 2012. $184 \mathrm{p}$.

[2] Delgado-Pertíñez M, Guzmán-Guerrero JL, Caravaca FP, Castel JM, Ruiz FA, González-Redondo P, et al. Effect of artificial vs. natural rearing on milk yield, kid growth and cost in Payoya autochthonous dairy goats. Small Rumin Res. 2009;84(1-3):108-15. doi: 10.1016/J.SMALLRUMRES.2009.06.014.

[3] Paez Lama S, Grilli D, Egea V, Fucili M, Allegretti L, Guevara JC. Rumen development and blood metabolites of criollo kids under two different rearing systems. Livest Sci. 2014;1(167):171-7. doi: https:// doi.org/10.1016/J.LIVSCI.2014.06.018.

[4] Ezema C. Probiotics in animal production: a review. J Vet Med Anim Heal. 2013;5(11):308-16.

[5] Brashears MM, Amezquita A, Jaroni D. Lactic acid bacteria and their uses in animal feeding to improve food safety. Adv Food Nutr Res. 2005;1(50):1-31. doi: https://doi.org/10.1016/S1043-4526(05)50001-9.

[6] Arowolo MA, He J. Use of probiotics and botanical extracts to improve ruminant production in the tropics: A review. Anim Nutr. 2018;4(3):241-9. doi: https://doi.org/10.1016/J.ANINU.2018.04.010.

[7] Adams MC, Luo J, Rayward D, King S, Gibson R, Moghaddam GH. Selection of a novel direct-fed microbial to enhance weight gain in intensively reared calves. Anim Feed Sci Technol. 2008;145(14):41-52. doi: https://doi.org/10.1016/J.ANIFEEDSCI.2007.05.035.

[8] Mudgal V, Baghel RPS. Effect of probiotic supplementation on growth performance of pre-ruminant buffalo (Bubalus bubalis) calves. Buffalo Bull. 2010;29(3):225-8.

[9] Magalhães VJA, Susca F, Lima FS, Branco AF, Yoon I, Santos JEP. Effect of feeding yeast culture on performance, health, and immunocompetence of dairy calves. J Dairy Sci. 2008;91(4):1497-509. doi: https:// doi.org/10.3168/jds.2007-0582
[10] Ayişiği K, Ataşoğlu C, Yaman-Yurtman I, Mendeş M, Pala A. Effect of probiotic supplementation shortly before and after weaning on growth of turkish saanen kids. Arch Anim Breed. 2005;48(6):60111. doi: https://doi.org/10.5194/aab-48-601-2005.

[11] Signorini ML, Soto LP, Zbrun MV, Sequeira GJ, Rosmini MR, Frizzo LS. Impact of probiotic administration on the health and fecal microbiota of young calves: A meta-analysis of randomized controlled trials of lactic acid bacteria. Res Vet Sci. 2012;93(1):250-8. doi: https://doi.org/10.1016/J. RVSC.2011.05.001.

[12] Chacón A. Comparación de la titulación de la acidez de leche caprina y bovina con hidróxido de sonio y cal común saturada. Agron Mesoam. 2006;17(1): 55-61.

[13] Saleem AM, Zanouny AI, Singer AM. Growth performance, nutrients digestibility, and blood metabolites of lambs fed diets supplemented with probiotics during pre- and post-weaning period. Asian-Australasian J Anim Sci. 2017;30(4):523-30. doi: https:// doi.org/10.5713/ajas.16.0691.

[14] Ataşoğlu C, Akbağ HI, Tölü C, Daş G, Savaş T, Yurtman IT. Effects of kefir as a probiotic source on the performance of goat kids. S Afr J Anim Sci. 2010;40(4):363-70.

[15] Daş G, Ataşoğlu C, Akbağ HI, Tölü C, Yurtman IY, Savaş T. Effects of kefir on coccidial oocysts excretion and performance of dairy goat kids following weaning. Trop Anim Health Prod. 2012;44(5):104955. doi: https://doi.org/10.1007/s11250-011-0039-3.

[16] Fomenky BE, Chiquette J, Bissonnette N, Talbot G, Chouinard PY, Ibeagha-Awemu EM. Impact of Saccharomyces cerevisiae boulardii CNCMI-1079 and Lactobacillus acidophilus BT1386 on total lactobacilli population in the gastrointestinal tract and colon histomorphology of Holstein dairy calves. Anim Feed Sci Technol. 2017;234:151-61. doi: https://doi. org/10.1016/J.ANIFEEDSCI.2017.08.019.

[17] Bomba A, Kravjanský I, Kaštel' R, Herich R, Juhásová Z, Čížek M, et al. Inhibitory effects of Lactobacillus casei upon the adhesion of enterotoxigenic Escherichia coli K99 to the intestinal mucosa in gnotobiotic lambs. Small Rumin Res. 1996;23(23):199-206. doi: https://doi.org/10.1016/S09214488(96)00905-4.

[18] Bomba A, Nemcová R, Gancarcíková S, Herich R, Kastel R. Potentiation of the effectiveness of lactobacillus casei in the prevention of $E$. coli induced diarrhea in conventional and gnotobiotic pigs. Adv Exp Med Biol. 2000;473:185-90.

[19] Krehbiel CR, Rust SR, Zhang G, Gilliland SE. Bacterial direct-fed microbials in ruminant diets: Per- 
formance response and mode of action. J Anim Sci. 2003;81(14_suppl_2):E120-32.

[20] Le OT, Dart PJ, Harper K, Zhang D, Schofield B, Callaghan MJ, et al. Effect of probiotic Bacillus amyloliquefaciens strain $\mathrm{H} 57$ on productivity and the incidence of diarrhoea in dairy calves. Anim Prod Sci. 2017;57(5):912-9. doi: https://doi.org/10.1071/ AN15776.

[21] Renaud DL, Kelton DF, Weese JS, Noble C, Duffield TF. Evaluation of a multispecies probiotic as a supportive treatment for diarrhea in dairy calves: A randomized clinical trial. J Dairy Sci. 2019;102(5):4498-4505. doi: https://doi.org/10.31 68/JDS.2018-15793.

[22] Allen SJ, Martinez EG, Gregorio G V, Dans LF. Probiotics for treating acute infectious diarrhoea. Cochrane Database of Systematic Reviews. John Wiley \& Sons, Ltd; 2010 nov. doi: https://doi. org/10.1002/14651858.CD003048.pub3.
[23] Ayala-Monter MA, Hernández-Sánchez D, González-Muñoz S, Pinto-Ruiz R, Marinez-Aispuro JA, Torres-Salado N, et al. Growth performance and health of nursing lambs supplemented with inulin and Lactobacillus casei. Asian-Australasian J Anim Sci. 2019; 32(8):1137-1144. doi: https://doi. org/10.5713/ajas.18.0630.

[24] Kara C, Orman A, Gencoglu H, Kovanlıkaya A, Meral Y, Cetin I, et al. Effects of inulin supplementation on selected faecal characteristics and health of neonatal Saanen kids sucking milk from their dams. animal. 2012;6(12):1947-54. doi: https://doi. org/10.1017/S1751731112000900. 